\title{
Increased conductivity of a hole transport layer due to oxidation by a molecular nanomagnet
}

\author{
S. Cheylan, ${ }^{1, a)}$ J. Puigdollers, ${ }^{2}$ H. J. Bolink, ${ }^{3}$ E. Coronado, ${ }^{3}$ C. Voz, ${ }^{2}$ R. Alcubilla, ${ }^{2}$ and \\ G. Badenes ${ }^{1}$ \\ ${ }_{1}^{1}$ ICFO_Institut de Ciencies Fotoniques, Mediterranean Technology Park, 08860 Barcelona, Spain \\ ${ }^{2}$ Departamento de Ingeniería Electrónica, Universidad Politécnica de Cataluña, Campus Nord Edifici C4, \\ c/ Jordi Girona 1-3, 08034 Barcelona, Spain \\ ${ }^{3}$ ICMol-Instituto de Ciencia Molecular, Universidad de Valencia, P.O. Box 22085, 46071 Valencia, Spain
}

(Received 7 December 2007; accepted 5 March 2008; published online 14 May 2008)

\begin{abstract}
Thin film transistors based on polyarylamine $\operatorname{poly}\left(N, N^{\prime}\right.$-diphenyl- $N, N^{\prime}$ bis(4-hexylphenyl)$\left[1,1^{\prime}\right.$ 'biphenyl]-4, $4^{\prime}$-diamine (pTPD) were fabricated using spin coating in order to measure the mobility of pTPD upon oxidation. Partially oxidized pTPD with a molecular magnetic cluster showed an increase in mobility of over two orders of magnitude. A transition in the mobility of pTPD upon doping could also be observed by the presence of a maximum obtained for a given oxidant ratio and subsequent decrease for a higher ratio. Such result agrees well with a previously reported model based on the combined effect of dipolar broadening of the density of states and transport manifold filling. (C) 2008 American Institute of Physics. [DOI: 10.1063/1.2917304]
\end{abstract}

In recent years, there have been increasing interests and research activities in the field of organic light-emitting devices (OLEDs). The promise of the use of this technology in flat panel displays through the fabrication of low-cost and flexible electronic devices has been the main driving force. Although enormous progress has been achieved in improving the luminance efficiency, color fidelity, and device lifetime, there is a continuous effort for a better stability of the device toward commercialization. Interface engineering between the electrodes and the emitting layer is important for the improvement of the device lifetime as well as luminous efficiency in OLEDs and has lead to the fabrication of multilayer devices, with charge transport and charge injection layer (HIL) deposited by vacuum sublimation technique. ${ }^{1,2}$ In terms of solution processable OLEDs, such as polymer based light emitting devices (PLEDs), the use of a PEDOT-PSS layer as the HIL is well known to improve the hole injection from the indium tin oxide (ITO) as well as planarizing the ITO surface and has been well studied. ${ }^{3,4}$ Although it has shown improved device performance such as lower operational voltage and higher luminance efficiency, other problems render it not suitable for device stability. For example, it is found that the ITO/PEDOT-PSS interface is not stable due to the etching of ITO by the strong acidic nature of PSS. ${ }^{5}$ Therefore, at this stage of PLED development, the search for other better solution processable HIL material is needed and it is much required to modify the HIL to further improve the device lifetime as well as its efficiency. Following such an approach, recent results by Bolink et al. ${ }^{6}$ have shown that a solution processable HIL material can be used for PLEDs and showed improved performance as when using PEDOT-PSS. Such HIL material is based on a polyarylamine poly $\left(N, N^{\prime}\right.$-diphenyl- $N, N^{\prime}$ bis(4-hexylphenyl)-[1,1'biphenyl]-4,4'-diamine (pTPD) which was

\footnotetext{
a) Author to whom correspondence should be addressed. Electronic mail: stephanie.cheylan@icfo.es
}

doped with a molecular magnet and showed tunable conductivity depending on the level of doping. The diamine derivative pTPD resembles very closely to the well known and intensively used organic molecule $N, N^{\prime}$-diphenyl- $N$, $N^{\prime}$ bis(3-methylphenyl)-[1, $1^{\prime}$ biphenyl]-4, $4^{\prime}$-diamine (TPD), while being processable by the spin coating technique and showing a lower conjugation length. In pTPD, the arylamine units are chemically linked to each other, however, due to the angle between the adjacent phenyl rings, the conjugation is limited and does only extend slightly along the chain direction. However, such a slight extended conjugation can result in slightly increased hole mobilities than in the case of TPD. Mobilities of the order of $10^{-5} \mathrm{~cm}^{2} / \mathrm{V} \mathrm{s}$ and 2.4 $\times 10^{-5} \mathrm{~cm}^{2} / \mathrm{V} \mathrm{s}$ can be obtained usually for spin coated TPD and pTPD-based films, respectively, showing slightly better mobility for pTPD material as expected. More recently, doping TPD or partially oxidizing it have shown possible enhancement of its charge mobility ${ }^{7,8}$ and resulted in OLED devices exhibiting very low driving voltages. ${ }^{9,10}$

In this study we chose to investigate the pTPD diamine derivative and determine its mobility in the normal and oxidized state using thin film transistors (TFT). We will show here that it is possible to improve quite significantly the charge mobility of pTPD, using a simple methodology. An enhancement of two orders of magnitude in the holes mobility of pTPD films have been achieved by oxidation of the pTPD solution with a molecular magnetic cluster $\left[\mathrm{Mn}_{12} \mathrm{O}_{12}\left(\mathrm{H}_{2} \mathrm{O}\right)_{4}\left(\mathrm{C}_{6} \mathrm{~F}_{5} \mathrm{COO}\right)_{16}\right]$.

pTPD was commercially available from American Dye Sources $(98 \%$ purity) with no further purification steps. A solution of $20 \mathrm{mg} / \mathrm{ml}$ of pTPD was made in chlorobenzene. $100 \mathrm{~nm}$ thin films were obtained by spin coating the filtered solutions at $1500 \mathrm{rpm}$. The molecular magnetic cluster $\left[\mathrm{Mn}_{12} \mathrm{O}_{12}\left(\mathrm{H}_{2} \mathrm{O}\right)_{4}\left(\mathrm{C}_{6} \mathrm{~F}_{5} \mathrm{COO}\right)_{16}\right]$, abbreviated as $\mathrm{Mn}_{12}$, was prepared using the ligand substitution method. ${ }^{11}$ The preparation of the $\mathrm{Mn}_{12}$ :pTPD solution in chlorobenzene was done by adding small amounts of $\mathrm{Mn}_{12}$ diluted dichlo- 


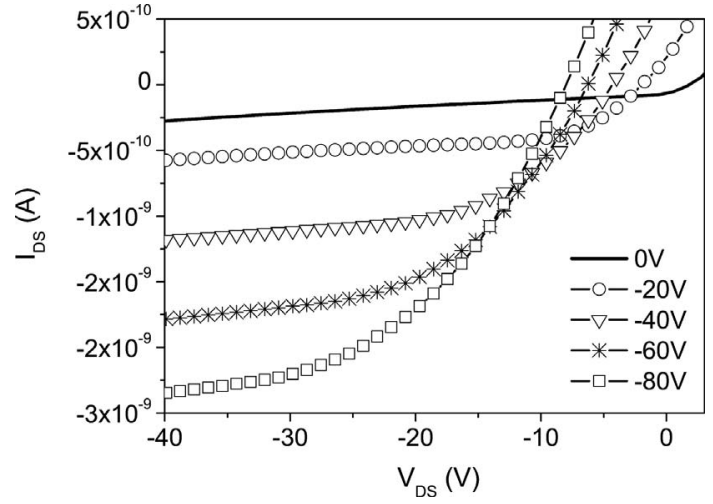

FIG. 1. Output characteristics of an OTFT device based on spin coated pTPD.

romethane solution to the pTPD solution. The pTPD films with varying amounts of $\mathrm{Mn}_{12}$ were spin coated from cholorobenzene solution onto $\mathrm{SiO}_{2}$ coated $\mathrm{Si}$ substrates. Highly doped $p$-type silicon wafers with $200 \mathrm{~nm}$ thick thermal $\mathrm{SiO}_{2}$ were used as the substrate of the bottom contact TFT device structure with gold drain and source electrodes. The electrical characterization of the TFTs was performed at room temperature in air using a 4156 Agilent semiconductor parameter analyzer in a slow scan mode.

Several doped pTPD-based OTFTs were fabricated and characterized in order to study their performance in function of the dopant level. All devices exhibited the operating characteristics of a $p$-channel field-effect transistor. In Fig. 1, the output characteristics of the pTPD OTFTs fabricated are shown. Figure 2(a) shows the $I_{\mathrm{DS}}$ plotted on a $\log$ scale as a function of $V_{\mathrm{GS}}$ for a $V_{\mathrm{DS}}$ of $-50 \mathrm{~V}$ (transfer characteristic). The field-effect mobility $(\mu)$ and the threshold voltage $V_{T}$ can be obtained from the saturation characteristics given by the equation $I_{D} \cong\left(\mu W C_{0} / 2 L\right)^{*}\left(V_{\mathrm{GS}}-V_{T}\right)^{2}$, where $W$ and $L$ are the channel width and length of the TFT and $C_{0}$ is the capacitance of the insulator per area unit of 1.725 $\times 10^{-8} \mathrm{~F} / \mathrm{cm}^{2}$. Figure 2(b) shows the curve of $\left|I_{\mathrm{DS}}\right|^{1 / 2}$ versus $V_{\mathrm{GS}}$ in the saturation regime used for the calculation. Mobilities $(\mu)$ were estimated to be around $2.4 \times 10^{-5} \mathrm{~cm}^{2} / \mathrm{V} \mathrm{s}$ for pTPD, with positive values of $1 \mathrm{~V}$ for the threshold voltage. The measured $\mu$ and $V_{T}$ values obtained for pTPD are consistent with results reported for OTFTs measured in ambient conditions and deposited on $\mathrm{SiO}_{2}$ dielectric layer (reported field-effect mobilities between $10^{-5}$ and $\left.10^{-4} \mathrm{~cm}^{2} / \mathrm{V} \mathrm{s}\right){ }^{12-14}$

The possibility of improving the mobility of such materials has been demonstrated and reported using various approaches. ${ }^{7,8,12-15}$ For example, it is possible to modify the polymer chain by anchoring end-capping groups, ${ }^{12}$ to use them as dopant for inert polymers such as polystyrene or polycarbonate, ${ }^{7,13,14}$ or to oxidize the arylamines with strong oxidant molecules such as $\mathrm{ClO}_{4}^{-}$and $\mathrm{AgSbF}_{6}{ }^{8,15}$ Recently, Bolink et al. ${ }^{6,16}$ have shown that pTPD properties can be modified by oxidizing it with a molecular magnetic cluster $\left[\mathrm{Mn}_{12} \mathrm{O}_{12}\left(\mathrm{H}_{2} \mathrm{O}\right)_{4}\left(\mathrm{C}_{6} \mathrm{~F}_{5} \mathrm{COO}\right)_{16}\right]$. They have shown improved conductivity as a function of the concentration of $\mathrm{Mn}_{12}$ used in the pTPD solution as well as improved OLED device performance using $\mathrm{Mn}_{12} / \mathrm{pTPD}$ as the hole-injection material. They demonstrated that the performance of a blue PLED
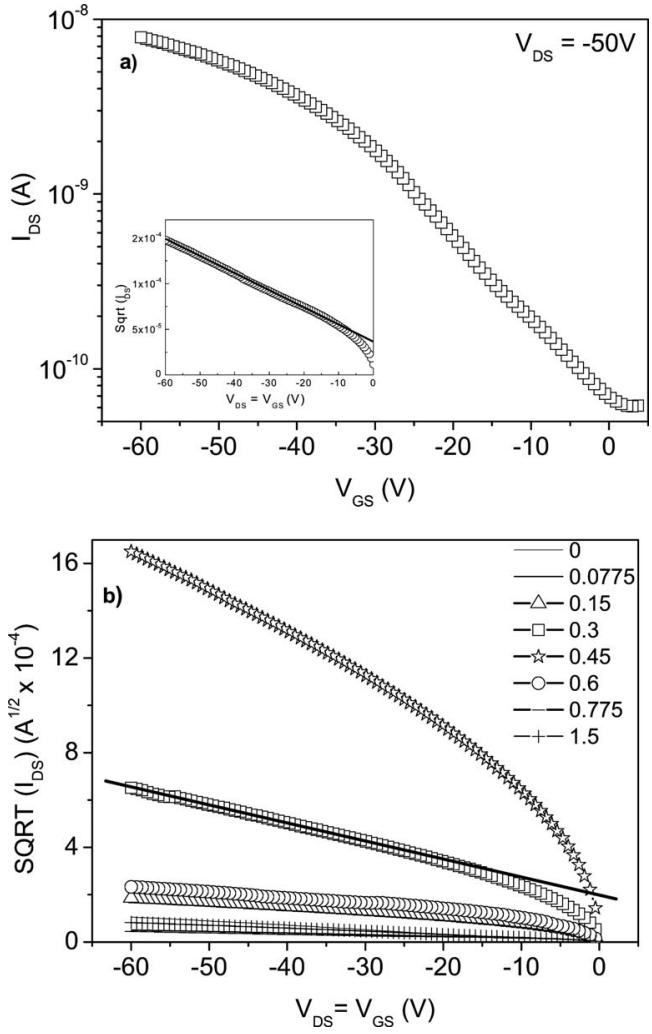

FIG. 2. (a) Transfer characteristics of a ( $\square$ ), for a $V_{D}$ of $-50 \mathrm{~V}$. The inset shows the saturation regime curve $(\bigcirc)$, from which the mobility and threshold voltage can be deduced. (b) Saturation regime curve as a function of the ratio of $\mathrm{Mn}_{12}$ to $\mathrm{pTPD}$ in the solution. A linear fit to the curves, used for calculating the mobility, is shown for the $\mathrm{Mn}_{12}$ ratio of 0.3 .

can be optimized by fine-tuning the composition of this hybrid hole-injection material, reaching efficacies of $3.5 \mathrm{~cd} / \mathrm{A}$ at $5000 \mathrm{~cd} / \mathrm{m}^{2}$. The conductivity of the hole-injection material is tunable and ranges from $10^{-8} \mathrm{~S} / \mathrm{cm}$ for the unoxidized pTPD to $0.01 \mathrm{~S} / \mathrm{cm}$ at relatively low levels of oxidation. ${ }^{16}$ Following these previous results, pTPD was oxidized by $\mathrm{Mn}_{12}$, using a wide range of concentrations and TFTs were fabricated based on such solutions in order to obtain values for the mobility of the oxidized pTPD. The mobilities of oxidized arylamine materials are not a trivial thing to determine as no precise models exist to deduce them from direct current current-voltage characteristics. In fact, the partial oxidation of arylamines, although resulting in an increase in conductivity, is still not completely understood. In their paper, Shen et al. ${ }^{15}$ describe the competing influence of at least three processes on the increase in conductivity. These are the increase of the width of the distribution of the density of states (DOS) due to an increased polarity by the partial oxidation, the Coulombic interaction between the delocalized charge on the arylamine and its counterion and the partial filling of the transport manifold. The first two processes in fact should decrease the mobility, whereas the latter is believed to be responsible for an increase in the materials mobility. Therefore, we attempted to determine the hole mobilities of partially oxidized pTPD via the prepartion of TFTs. Interestingly we were able to deduce the hole mobilities as a function of oxidation ratio. Their dependence on the proportion of $\mathrm{Mn}_{12}$ present in the solution is shown in Fig. 3. As 


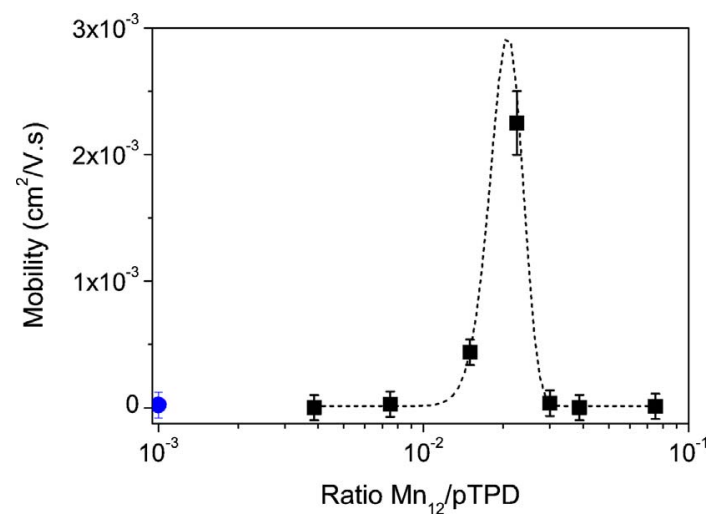

FIG. 3. (Color online) Field-effect mobility of pTPD: $\mathrm{Mn}_{12}$ as a function of the ratio of $\mathrm{Mn}_{12}$ to $\mathrm{pTPD}$ in the solution. The mobility of pTPD alone was measured to be $\mu=2.5 \times 10^{-5} \mathrm{~cm}^{2} / \mathrm{V} \mathrm{s}$ and is represented as a round dot on the graph. A Gaussian fit is added just for an indication of the dependence behavior.

mentioned previously, in this work, the mobility of pTPD alone was found to be $2.4 \times 10^{-5} \mathrm{~cm}^{2} / \mathrm{V} \mathrm{s}$. As can be seen, when a small amount of $\mathrm{Mn}_{12}$ is introduced in the pTPD solution (ratio of $\mathrm{Mn}_{12}$ /pTPD less than 0.01), an initial decrease in mobility can be observed. As the proportion of $\mathrm{Mn}_{12}$ is increased, so is the mobility. A maximum mobility of $2.4 \times 10^{-3} \mathrm{~cm}^{2} / \mathrm{V} \mathrm{s}$ is obtained for a ratio of $\mathrm{Mn}_{12} / \mathrm{pTPD}$ of 0.0225 , which consists of an improvement of about two orders of magnitude compared to pTPD alone. Then as the proportion of $\mathrm{Mn}_{12}$ is further increased, the mobility decreases again down to $2.4 \times 10^{-5} \mathrm{~cm}^{2} / \mathrm{V} \mathrm{s}$ for ratios of $\mathrm{Mn}_{12} / \mathrm{pTPD}$ above 0.03 . Up to this high ratio of dopant, a TFT behavior could be observed and therefore meaningful values for the mobility could be obtained. The nonlinear behavior of the mobility of the oxidized pTPD as a function of the dopant ratio exactly follows the prediction made from the model put forward by Shen et al., ${ }^{15}$ which is due to a combined effect of dipolar broadening of the DOS and manifold filling. The initial decrease in mobility can be explained by the increase in the broadening of the transport manifold due to the enhanced disorder coming from the dopant. At higher oxidation ratios, the manifold filling becomes more important and the mobility increases until the polarity gets so high for a higher oxidation ratio that Coulombic effects appear and reduce the mobility again. Such nonlinear behavior is quite common in organic blends, where an optimized system (in terms of luminescence efficiency, conductivity, mobility, etc.) is obtained for a certain concentration of dopant and decreases for further increase in concentration. This can also be clearly seen in the transition observed in the device operation when using such material for the HIL. ${ }^{16}$ It goes from a hole-limited device (ratio of $\mathrm{Mn}_{12}$ / pTPD lower than 0.01) to a charge balance device (ratio 0.01) and eventually to a device which is electron limited (ratio above 0.01). ${ }^{16}$

Finally, it is important to report that significant improvement in the pTPD mobility has previously been reported using other dopants, such as polyhedral oligomeric silsesquioxanes ${ }^{11}$ (POSS), where an increase in mobility of one order of magnitude was also obtained (around $10^{-2} \mathrm{~cm}^{2} / \mathrm{V} \mathrm{s}$ ). In that case, the mobility improvement was only observed when the POSS was added to the main chain of the polymer via chemical synthesis. The pTPD: $\mathrm{Mn}_{12}$ blend offers a simpler technique to obtain improved mobilities compared to the latter one. The pTPD polymer really presents attractive properties for device applications such as a spin coating technology, nice uniform films, and mobility (and conductivity) that can be improved to reach up to $10^{-3}-10^{-2} \mathrm{~cm}^{2} / \mathrm{V} \mathrm{s}$ by using either organic or inorganic dopants.

In conclusion, we have shown that the mobility of pTPD can be improved by two orders of magnitude by oxidizing the solution with a molecular magnetic cluster $\mathrm{Mn}_{12}$. Such enhanced mobility is not linear with the proportion of $\mathrm{Mn}_{12}$ present in the solution and a maximum was obtained for a ratio of $\mathrm{Mn}_{12} / \mathrm{pTPD}$ of 0.0225 . It can be explained by a combined effect of dipolar broadening of the DOS due to the increased disorder coming from the dopant and the transport manifold filling. This model was already put forward to explain the variation of the conductivity of the oxidized polymer in function of the oxidant ratio. Such results are important in order to understand the phenomena at stake in the transport mechanism for doped or oxidized polymers. Furthermore, a polymer such as pTPD really presents attractive properties for device applications such as spin coating technology, nice uniform films, a lower conjugation length than TPD, and mobility (and conductivity) that can be improved to reach up to $10^{-3}-10^{-2} \mathrm{~cm}^{2} / \mathrm{V}$ s by using either organic or inorganic dopants.

S.C. and H.J.B. acknowledge support from the Spanish Ministry of Education and Science through the Ramon y Cajal program. This work was carried out with the financial support of the Spanish Ministry of Education and Science through Grant Nos. TEC2006-10665/MIC and ConsoliderIngenio CSD2007-00007.

${ }^{1}$ H. Kanno, N. C. Giebink, Y. Sun, and S. R. Forrest, Appl. Phys. Lett. 89, 023503 (2006).

${ }^{2}$ Y. Kim, E. Oh, H. Lim, and C.-S. Ha, Appl. Phys. Lett. 88, 043504 (2006). ${ }^{3}$ R. E. Gill, P. van de Weijer, C. T. H. Liedenbaum, H. F. M. Schoo, A. Berntsen, J. J. M. Vleggaar, and R. J. Visser, Opt. Mater. 12, 183 (1999). ${ }^{4}$ Y. Cao, G. Yu, C. Zhang, R. Menon, and A. J. Heeger, Synth. Met. 87, 171 (1997).

${ }^{5}$ M. P. de Jong, L. J. van Ijzendoorn, and M. J. A. de Voigt, Appl. Phys. Lett. 77, 2255 (2000).

${ }^{6}$ H. J. Bolink, L. Cappelli, E. Coronado, and I. Recalde, Adv. Mater. (Weinheim, Ger.) 18, 920 (2006).

${ }^{7}$ S. H. Kim, Y. S. Yang, J. H. Lee, J.-I. Lee, H. Y. Chu, H. Lee, J. Oh, L.-M. Do, and T. Zyung, Opt. Mater. 21, 439 (2003).

${ }^{8}$ A. Troup, J. Mort, S. Grammatica, and D. J. Sandman, J. Non-Cryst. Solids 35-36, 151 (1980).

${ }^{9}$ T. Ito, J. Asaka, K. L. Thanh Dao, and J. Kido, Polym. Adv. Technol. 16, 559 (2005).

${ }^{10}$ M. Pfeiffer, K. Leo, X. Zhou, J. S. Huang, M. Hofmann, A. Werner, and J. Blochwitz-Nimoth, Org. Electron. 4, 89 (2003).

${ }^{11}$ H. J. Eppley, H. L. Tsai, N. D. Vries, K. Folting, G. Christou, and D. N. Hendrickson, J. Am. Chem. Soc. 117, 301 (1995).

${ }^{12}$ W. W. Zhu, S. Xiao, and I. Shih, Appl. Surf. Sci. 221, 358 (2004).

${ }^{13}$ F. Khan and P. R. Sundararajan, Org. Electron. 7, 410 (2006).

${ }^{14}$ S. R. Mohan and M. P. Joshi, Solid State Commun. 139, 181 (2006).

${ }^{15}$ Y. Shen, K. Diest, M. H. Wong, B. R. Hsieh, D. H. Dunlap, and G. G. Malliaras, Phys. Rev. B 68, 081204 (2003).

${ }^{16}$ H. J. Bolink, E. Coronado, A. Forment-Aliaga, and C. J. Gómez-García, Adv. Mater. (Weinheim, Ger.) 17, 1018 (2005). 